\title{
Mix design for self-compacting palm oil clinker concrete based on particle packing
}

\author{
Jegathish Kanadasan, Hashim Abdul Razak
}

Self-compacting concrete (SCC) has progressively developed to become a concrete that is both efficient as well as providing a quality product without comprising its durability. Reduced labour needs, good surface finishing, extended workability and excellent hardened properties are some of the key elements of SCC. SCC is designed in a way that it will be able to flow under its own weight and produce good self compactability features without the need of any external vibration. The study of the particle packing (PP) gives a good understanding concerning the consumption of aggregate and paste volume for a given unit volume of concrete. Hence, optimisation of particle packing in SCC is vital to improve the flow behaviour and also enhance the hardened properties. Over the years, the application of lightweight self-compacting concrete (LSCC) has been progressing rapidly with proven versatility in the reduction of the dead load of the overall structure. The main concern of LSCC is the consumption of 1ightweight aggregate, which is usually lower in density coupled with high porosity.

The agricultural industry has been a mainstay of the Malaysian economy for the past two decades with millions of hectares being planted with oil palm, rubber, paddy, sugar cane, coconut and cocoa. The extraction of useful material from these plants generates various types and forms of waste material, which need to be disposed of appropriately. Generally, they comprise ash, grains, wastewater, shells and large combined chunks. Malaysia is one of the largest producers and exporters of palm oil products to various corners of the world. Plenty of waste by-products are collected throughout the palm oil processing phases. One of them is palm oil clinker (POC), which is obtained in large chunks during the oil palm shell and fibre incineration process. Nowadays, POC is disposed of by landfill. This not only causes soil pollution but also 
affects the ground water supply source. Therefore, the consumption of these abundantly available waste products in the construction industry will not only help to reduce the environmental problems but also provide an alternative to the diminishing natural aggregates. The sustainability of these aggregates, which is one of the major issues in the construction industry, can be addressed accordingly.

\section{Background studies}

Concrete generally comprises aggregate, cementitious materials and some chemical admixtures. The bond or interaction between them plays a vital part in determining most of the fresh and hardened properties of concrete. The proper arrangement of particulates in concrete not only enhances the interlocking effects between them but also promotes some of the key hardening and durability features. Hence, optimisation of PP in concrete would promote enhanced performance due to the dense arrangement of the structure. Increasing the packing density of the aggregate can reduce the binder content as well as decrease the cost of the concrete [1]. It has also been reported that improved aggregate packing has the potential to enhance the properties of concrete in terms of strength, modulus of elasticity, creep and shrinkage [2]. The interlocking effect and interaction between the aggregate is also closely related to the aggregate size whereby addition of aggregates greater than $2.36 \mathrm{~mm}$ induces good aggregate contact [3].

Kwan and Fung [4] explained that increasing the packing density has a significant improvement on the rheological behaviour of cement paste and mortar. While Jalal et al. [5] reported that the increase in paste volume, due to the additional binder content, may enhance the rheological properties of SCC. Girish et al. [6] reported that an increase in paste volume elevates the slump flow values generally for varying water content. While on the other hand, when utilising two types of lightweight aggregate with different densities for SCC production, it was found that the lighter ones gives larger slump flow [7]. Incorporation of lightweight aggregate also has the capability to reduce the viscosity of the mix as the movement of aggregates gets easier through the mortar phase $[8,9]$.

Research has diverged from the production of ordinary SCC to the utilisation of abundantly available waste materials as aggregate replacement and binder substitution. As the world is moving towards the concept of 3R (Recycle, Reuse, Reduce) and a sustainable 
environment, the use of these waste materials not only helps to save the environment but also preserves the depletion of natural resources. Plenty of waste products from the agricultural industry are currently utilised for the production of normal concrete and SCC, such as rice husk ash (RHA), sawdust ash (SDA), palm oil fuel ash (POFA), rubber aggregate and bagasse ash. Memon et al. [10], found that by incorporating 10\% RHA, the strength of SCC improves with the increase in superplastisizer (SP) content as an effect of the enhanced self-compacting behaviour. Furthermore, the addition of bagasse ash up to $30 \%$ produces concrete with a good compressive strength compared to control concrete [11]. The use of rubber aggregate produces SCC that is less prone to cracking due to lesser restrained shrinkage [12]. Elinwa et a1. [13] reported that SDA reacts during the cement hydration process to produce gel that enhances the overall structure of the concrete. In addition, other waste by-products that are used as aggregate as well as binder replacements in SCC production include crumb rubber, recycled glass (RG), marble dust (MD), rubble powder and waste liquid crystal glass (LCD). Topcu et al. [14] reported that utilising MD at $200 \mathrm{~kg} / \mathrm{m} 3$ can be considered as maximum and ideal to produce significant positive effect on both the fresh and hardened properties of SCC. SCC incorporating LCD glass sand at 30\% substitution level produced higher ultrasonic pulse velocity (UPV) and electrical resistance values compared to control mix [15]. With the addition of fine recycled concrete aggregate, the resistance of SCC against chloride ion penetration increases [16].

Self-compacting rubberised concrete (SCRC) shows a lower Young' s modulus of elasticity (E) for all mixes but their flexural toughness improves [17]. General1y, the use of quarry waste limestone powder in SCC at constant cement content decreases the SP content and enhances the 28 days compressive strength [18]. Furthermore, the use of waste concrete powder (WCP) as a replacement for the cement generally reduces the viscosity of the paste and compressive strength compared to the one with only ordinary Portland cement (OPC) [19]. An investigation carried out for 650 days to study the chloride induced corrosion resistance in normal concrete with cement content substituted with volcanic ash (VA) and volcanic pumice powder (VPP) indicate that with $20 \%$ replacement levels, the concrete performs better in resisting corrosion. $[20]$. 
This study is divided into two different phases. The first phase comprised developing methods to study the PP for a combination of POC aggregate and ordinary aggregate. The techniques were modified to cater for the porous and lightweight physical characteristics of POC. The second phase of the experimental work consisted of developing a mix proportion for SCC, incorporating the PP results. Selected mix proportions were verified by preparing the actual POC SCC and their performance was evaluated for fresh and hardened properties.

The mix design for SCC proposed earlier by various researchers differs considerably with regards to the ordinary concrete. Currently, there are no fixed methods or definite values or factors available to supplement the SCC design process. Okamura and Ouchi [21] proposed a mechanism for achieving self-compactability by limiting the aggregate content, lowering the water powder ratio and using SP. Generally, the method involved designing a mix proportion considering the coarse aggregate phase and mortar phase.

Initially the coarse aggregate level was kept at $50 \%$ of the total concrete volume and the fine aggregate ratio at $40 \%$ of the mortar volume.

Then, the water/powder and SP dosage was determined to achieve the required flow of SCC. Trials were carried out by testing the mix design against the SCC properties compliance test.

Su et a1. [22] proposed a new methodology to develop mix design for SCC through integration of the packing factor (PF) between the aggregate in different states. They suggested the PF could be defined as the ratio of aggregate in the packed state to that of the loose state. Higher values of PF indicate a higher level of aggregate content with a reduction in mortar volume. While, conversely, lower values of PF relate to a higher binder content. Hence, the selection of optimum PF is vital to create a balance between cost and the concrete properties. In addition, in their study Choi et al. [23] modified the Su et al. [22] mix design process by attempting a different view of the packing model. They performed the determination of PF separately for fine and coarse aggregate. The PF obtained is related through the volume ratio of fine to total aggregate (S/a). Furthermore, Dinakar et a1. [24] designed SCC for ground granulated blast furnace slag (GGBS) applying the efficiency concept methodology whereby in their study the efficiency of slag activity was studied at varying replacement levels and the slag replacement was chosen according to the strength required. 
The cement content, as proposed by the method was altered in this mix design by the trial and mix process. A suitable cement content for a fixed aggregate ratio was studied by varying the POC substitution ratio and fine/total aggregate (F/A) ratio. In addition, the PP method, which focuses on aggregate packing and paste volume, were integrated into the study to form the mix proportions. Throughout the process of mix design, POC powder was utilised as additional binder content rather than as the percentage replacing cement to enhance the SCC characteristics.

Full text available at :

http://www.sciencedirect.com/science/article/pii/S0261306913010297

http://ac.els-cdn.com/S0261306913010297/1-s2.0-S0261306913010297-main.pdf? tid=0b7297f0-781f-11e3b904-00000aab0f01\&acdnat $=1389156228$ ce1d11c99ce21efab7f2b4b4bcf438be 